\title{
Reduction of systolic and diastolic dysfunction by retrograde coronary sinus perfusion during off-pump coronary surgery
}

Manuel Castellá, MD

Gerald D. Buckberg, MD

From the Department of Surgery, Division of Cardiothoracic Surgery, University of California, Los Angeles School of Medicine, Los Angeles, Calif.

Read at the Eighty-third Annual Meeting of The American Association for Thoracic Surgery, Boston, Mass, May 4-7, 2003.

Received for publication May 2, 2003; revisions requested Aug 27, 2003; accepted for publication Sept 8, 2003.

Address for reprints: Gerald D. Buckberg, MD, Division of Cardiothoracic Surgery, 62-258 Center for the Health Sciences, Los Angeles, CA 90095-1701 (E-mail: gbuckberg@mednet.ucla.edu).

J Thorac Cardiovasc Surg 2004;127:1018-25

$0022-5223 / \$ 30.00$

Copyright (C) 2004 by The American Association for Thoracic Surgery

doi:10.1016/j.jtcvs.2003.09.013
Objectives: We evaluated the protective effects of retrograde coronary sinus perfusion to offset potential systolic and diastolic dysfunction (myocardial stunning) after temporary regional ischemia needed for off-pump coronary artery bypass grafting.

Methods: Twenty Yorkshire-Duroc pigs $(31.8 \pm 3.9 \mathrm{~kg})$ underwent 15 minutes of mid-left anterior descending coronary artery ischemia in the beating heart. In 8 pigs, no protective measures were used. In 12 pigs, an aorta-coronary sinus shunt (with conventional cannulas) allowed retrograde perfusion during temporary ischemia; in 6 of these pigs, no leakage to the right atrium was ensured. Regional endocardial contraction was measured with sonomicrometer crystals. Systolic dysfunction (impaired regional shortening), diastolic dysfunction (contraction extending into early diastole), and coronary sinus nitric oxide and endothelin-1 levels were recorded.

Results: Before ischemia, contraction did not extend into the diastolic interval. During ischemia, paradoxic bulging occurred in all hearts except in the occlusive coronary sinus shunt group $(16 \% \pm 6 \%$ of baseline, $P<.01)$. Sixty minutes after ischemia, systolic segment shortening recovered $36 \% \pm 24 \%$ without retrograde perfusion versus $56 \% \pm 20 \%$ and $61 \% \pm 14 \%$ with coronary sinus shunting $(P<$ $.05)$. Diastolic dysfunction (as percentage of diastolic time in contraction) was $38 \%$ $\pm 16 \%$ in the nontreated group versus $22 \% \pm 22 \%$ and $9 \% \pm 9 \%(P<.05)$ after shunting and occlusive shunting, respectively. This correlated with a left ventricular end-diastolic pressure increase of $4 \mathrm{~mm} \mathrm{Hg}$ in the ischemic group versus no change in the retrograde perfusion groups. Nitric oxide decreased $15 \%$ without shunting and increased $8 \%$ after occlusive coronary sinus shunting $(P<.05)$.

Conclusions: Retrograde coronary sinus perfusion during simulated off-pump coronary revascularization diminishes systolic and diastolic dysfunction. An aorticcoronary sinus shunt is a rapid, recognized approach that can improve myocardial muscle and endothelial safety during off-pump coronary artery bypass grafting.

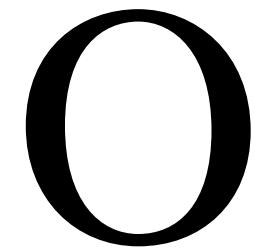

ff-pump coronary artery bypass (OPCAB) surgery has progressively become a standard procedure in many centers. Nevertheless, such temporal, unprotected myocardial ischemia may produce myocardial stunning, ${ }^{1,2}$ and brief periods of ischemia during multiple grafting may lead to perioperative heart insufficiency. Consequently, the OPCAB counterpart exists experimentally because a temporary period of ischemia, corresponding to the 15 -minute interval sometimes needed to construct a distal graft, causes both systolic and diastolic dysfunction. ${ }^{1}$ Furthermore, myocardial dysfunction may increase in an additive manner $^{2}$ with subsequent bouts of ischemia. Conversely, maintaining coronary flow throughout the coronary ischemic time can ameliorate these effects. One approach is the insertion of intracoronary shunts, but their flow is limited by proximal stenosis and they may produce initial injury when placed in damaged vessels. ${ }^{3}$ 


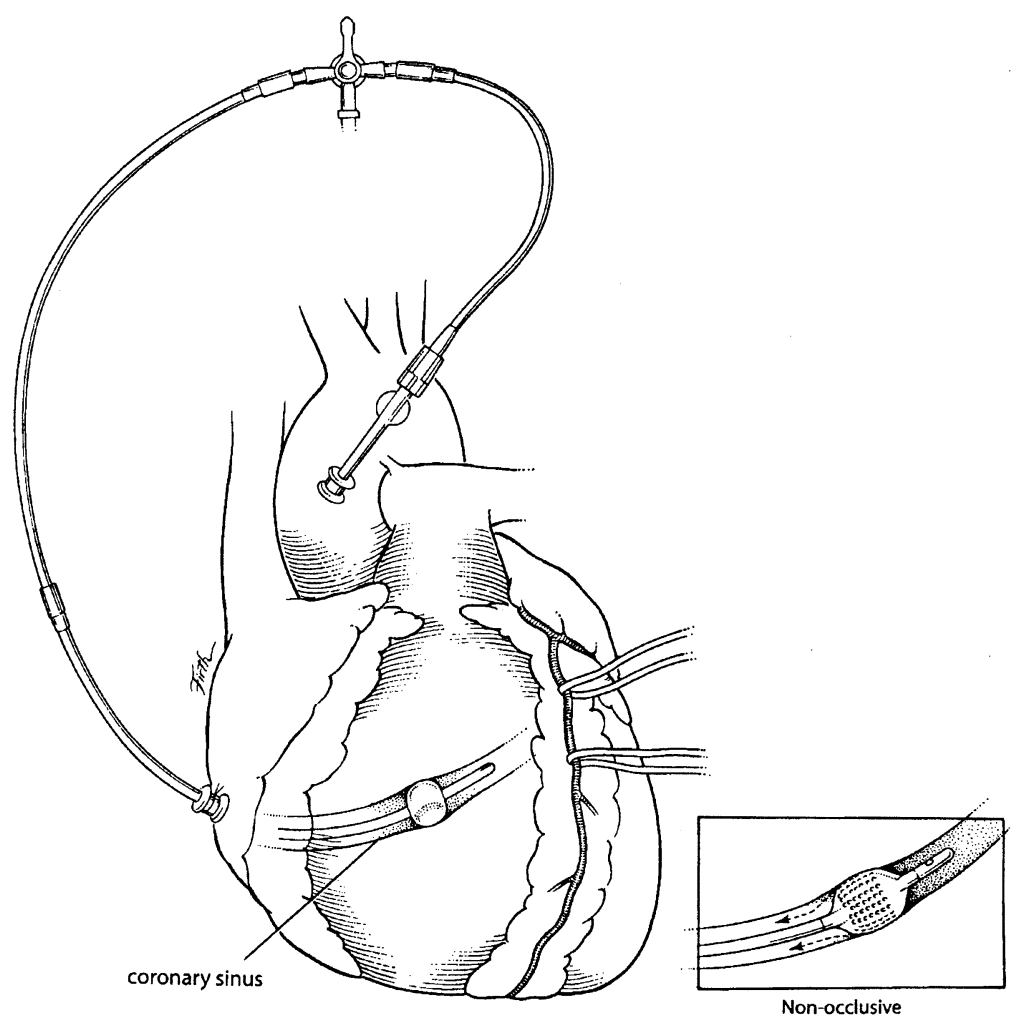

Figure 1. Aorta to CS shunt, connecting an antegrade to retrograde cardioplegia cannulas through a Luer connection. Two different retrograde cannulas were used with self-inflating textured balloon and manually inflating balloon.

Another alternative is retrograde coronary perfusion, because coronary sinus (CS) cannulation is routinely used in on-pump coronary artery bypass grafting (CABG), and early promising clinical results have been reported in highrisk patients. ${ }^{4}$

This study tested whether continuous retrograde coronary perfusion during a simulated 15 -minute time frame (to match the interval for a distal graft by simple connection of antegrade and retrograde CS cannulas) could prevent resultant systolic and diastolic dysfunction.

\section{Material and Methods}

All animals received humane care in compliance with the "Principles of Laboratory Animal Care," formulated by the Institute of Laboratory Animal Resources, and the "Guide for the Care and Use of Laboratory," prepared by the National Institutes of Health (Publication No. 86-23, revised 1985).

Twenty-two Yorkshire-Duroc pigs $(31.8 \pm 3.9 \mathrm{~kg})$ of either sex were premedicated (ketamine $15 \mathrm{mg} / \mathrm{kg}$, diazepam $0.5 \mathrm{mg} / \mathrm{kg}$ intramuscularly) and anesthetized (pentobarbital $30 \mathrm{mg} / \mathrm{kg}$ intravenously and subsequent bolus injections of sodium pentobarbital). Support with a volume-controlled ventilator (Servo 900C, Siemens-Elema, Solna, Sweden) was started after tracheostomy and endotracheal intubation. The femoral artery and vein were cannulated, and arterial blood gases were measured to keep oxygen tension, carbon dioxide tension, and $\mathrm{pH}$ values within the normal range. A balloon-tipped catheter (Model 132F5, Baxter Healthcare Corp, Irvine, Calif) was advanced into the pulmonary artery through a jugular vein to measure pulmonary artery and wedge pressures, and cardiac output was measured by the thermodilution technique.

The pericardium was incised after a median sternotomy, and a solid-state pressure transducer-tipped catheter (Model MPC-500, Millar Instruments, Inc, Houston, Tex) was inserted through the apex to monitor left ventricular pressure (LVP). A segment of the left anterior descending (LAD) coronary artery, distal of the first major diagonal branch, was circumferentially dissected. A 3-0 Prolene suture in between 2 Teflon pledgets was placed surrounding the LAD, leaving the LAD coronary vein free. A cardioplegia cannula was inserted into the ascending aorta, and a retrograde cardioplegia cannula was introduced transatrially into the CS. In the treated groups, both cannulas were connected by a $1 / 4$-inch polyvinyl chloride tube through Luer connections. The line was clamped until the start of myocardial ischemia (Figure 1).

\section{Experimental Protocol}

Animals were divided with the following groups:

1. Control no ischemia. Two normal hearts undergoing full catheterization did not have LAD ischemia.

2. Unprotected ischemia. Eight pigs underwent 15 minutes of LAD occlusion followed by 60 minutes of reperfusion. 
3. Coronary sinus shunt groups. Twelve pigs underwent 15 minutes of LAD occlusion. Retrograde flow into the CS was performed by connecting a retrograde cannula to the aortic perfusion cannula only during the ischemic time. Normal antegrade flow through the aorta was used during reperfusion.

3.1. Non-occlusive CS shunt. In 6 pigs, a 14F self-inflated, balloon-tipped retrograde cannula (Edwards RC-014T, Salt Lake City, Utah) was transatrially placed in the CS and connected to the aortic cannula, allowing a flow of 250 to $300 \mathrm{~mL} / \mathrm{min}$ at normal range aortic pressures. The serrated balloon surface allowed mild leakage back into the right atrium.

3.2. Occlusive CS shunt. Six hearts underwent the same ischemic protocol, using a 14F pressured balloon-tipped retrograde cannula (Edwards RC-014 MIBB, Salt Lake City, Utah). CS pressures were monitored when the shunt was open to ensure occlusion from right atrium.

\section{Hemodynamic Measurements}

LVP was electronically differentiated to obtain $\mathrm{dP} / \mathrm{dt}$, the first derivative of LVP. The cardiac cycle was defined automatically using $\mathrm{dP} / \mathrm{dt}$. Systole began 40 milliseconds before peak positive $\mathrm{dP} / \mathrm{dt}$ and ended 20 milliseconds before peak negative $\mathrm{dP} / \mathrm{dt}$, as previously defined by other groups. 5,6

Two 2-mm ultrasonic microtransducer crystals (Sonometrics, London, Ontario, Canada) were placed in the endocardial side of the anterior free wall of the left ventricle supplied by the LAD artery. Adequacy of LAD crystal placement was checked with 40 -second LAD occlusion during the equilibration period. A control measurement consisted of crystals in the muscle supplied by the left circumflex coronary artery. Data were recorded digitally with acquisition hardware and software (Sonometrics). Systolic shortening was defined as the change in segment length between the start and end of systole. Diastole was defined as the time that LVP decreased below aortic diastolic pressure or the origin of the isovolumetric interval. The percentage of systolic shortening was calculated as the ratio in percent between systolic shortening and end-diastolic length. Contraction during the first part of diastole was analyzed by the percentage of time of diastole in which contraction is in effect.

Cardiac output was determined by 4 central venous injections of $3 \mathrm{~mL}$ of $4^{\circ} \mathrm{C}$ saline solution. Left ventricular stroke work index (LVSWI) was calculated by the following formula: LVSWI = (mean arterial pressure - left auricular pressure) $\times$ cardiac output $\times 0.0136 \times$ heart rate $^{-1} \times$ weight $^{-1}$.

\section{CS Blood Analysis}

Myocardial injury was determined by analyzing CS blood samples taken 5 minutes before and 30 and 60 minutes after the onset of myocardial ischemia.

As a marker of oxidant-mediated lipid peroxidation, conjugated diene (CD) levels were determined spectrophotometrically in CS plasma after chloroform-methanol 2:1 ( $\mathrm{vol} / \mathrm{vol})$ extraction as previously described. ${ }^{7} \mathrm{CD}$ concentration was expressed as absorbance (A) at a wavelength of $240 \mathrm{~nm}$ per $0.5 \mathrm{~mL}$ plasma.

Myocardial damage was determined by measuring plasmatic creatine kinase fraction MB (units/liter) by an ultraviolet-spectro- photometric method (Sigma Chemical Co, St Louis, Mo), as recommended by the German Society for Clinical Chemistry.

Nitric oxide (NO) (micromoles/liter) was determined as its spontaneous oxidation products, nitrite and nitrate, which were converted to NO and quantitated by a chemiluminescence assay using a nitrogen oxides analyzer (DASIBI Environmental Corp, Model 2108, Glendale, Calif).

Endothelin (ET)-1 levels (picograms/milliliter) were determined after sample purification (Ethyl C2 Amprep minicolumns, Amersham Pharmacia Biotech, Piscataway, NJ) by an enzyme immunometric assay (ACETM EIA kit, Cayman Chemical Company, Ann Arbor, Mich) based on a double-antibody "sandwich" technique.

\section{Myocardial Biopsy}

At the end of the experiment, the pigs were killed by bolus injections of pentobarbital $250 \mathrm{mg}$ followed 1 minute later by 15 $\mathrm{mL}$ of cold hyperkalemic blood $(\mathrm{KCl}, 30 \mathrm{mEq} / \mathrm{L})$. With the $\mathrm{LAD}$ and the ascending aorta crossclamped, $60 \mathrm{~mL}$ of methicillin blue were injected through the aortic root into the coronary arteries to stain the non-ischemic myocardium. The ischemic myocardium was calculated as the percentage of dry weight between ischemic and non-ischemic myocardium.

\section{Statistical Analysis}

Statistical analysis of data within and between groups was performed with multiple analysis of variance followed by the Student $t$ test with Tukey-Kramer correction for multiplicity. Data are expressed as mean $\pm \mathrm{SD}$.

\section{Results}

The anesthetic and surgical procedure, including manipulation of the LAD without ischemia, did not alter hemodynamics or biochemistry.

Fifteen minutes of mid-LAD occlusion produced an ischemic zone of $27 \% \pm 4 \%$ and $3 \% \pm 2 \%$ of left and right ventricles, respectively, without differences among groups. In unprotected ischemia, bulging of the anterior wall occurred within seconds after LAD clamping, and each pig had at least 1 episode of ventricular fibrillation successfully treated by $10 \mathrm{~J}$ defibrillation. A 5-mg lidocaine bolus injection was only used to prevent further fibrillation. However, 7 of the 8 pigs had ventricular fibrillation during reperfusion, despite the lidocaine supplement. Mean arterial and left atrial pressures remained constant during the procedure, but cardiac output decreased by $17 \% \pm 20 \%$ at 30 minutes and by $34 \% \pm 27 \%$ at 60 minutes, causing LVSWI to decrease to $67 \% \pm 26 \%$ and $58 \% \pm 18 \%$ of baseline in the postischemia period (Table 1). Left ventricle end-diastolic pressure (LVEDP) increased from $8 \pm 3 \mathrm{~mm} \mathrm{Hg}$ to $12 \pm 7$ $\mathrm{mm} \mathrm{Hg}$ during ischemia and remained high after 1 hour of reperfusion (12 $\pm 5 \mathrm{~mm} \mathrm{Hg}$ at 60 minutes).

Regional sonometric analysis of the anterior left ventricular free wall showed that all contraction occurred during systole, without contraction during diastole. With unprotected ischemia, systolic bulging immediately followed cor- 
TABLE 1. Hemodynamic parameters

\begin{tabular}{|c|c|c|c|c|}
\hline Parameter & Group & Baseline & $30 \mathrm{~min}$ & $60 \mathrm{~min}$ \\
\hline \multirow[t]{3}{*}{ HR (beats/min) } & Ischemia & $87 \pm 11$ & $97 \pm 26$ & $106 \pm 21$ \\
\hline & CS shunt & $98 \pm 20$ & $124 \pm 20$ & $112 \pm 14$ \\
\hline & Occlusive CS shunt & $99 \pm 16$ & $102 \pm 13$ & $101 \pm 16$ \\
\hline \multirow[t]{3}{*}{$\mathrm{CO}$ (L/min) } & Ischemia & $3.62 \pm 0.70$ & $2.93 \pm 0.38$ & $2.23 \pm 0.46$ \\
\hline & CS shunt & $2.98 \pm 0.43$ & $2.37 \pm 0.25$ & $2.13 \pm 0.14$ \\
\hline & Occlusive CS shunt & $4.08 \pm 0.51$ & $3.46 \pm 0.74$ & $3.00 \pm 0.61$ \\
\hline \multirow[t]{3}{*}{$\mathrm{MAP}(\mathrm{mm} \mathrm{Hg})$} & Ischemia & $78 \pm 11$ & $76 \pm 10$ & $80 \pm 8$ \\
\hline & CS shunt & $74 \pm 9$ & $84 \pm 12$ & $83 \pm 11$ \\
\hline & Occlusive CS shunt & $84 \pm 14$ & $87 \pm 14$ & $90 \pm 14$ \\
\hline \multirow[t]{3}{*}{ LAP $(\mathrm{mm} \mathrm{Hg})$} & Ischemia & $9 \pm 2$ & $9 \pm 3$ & $8 \pm 3$ \\
\hline & CS shunt & $9 \pm 2$ & $8 \pm 2$ & $9 \pm 2$ \\
\hline & Occlusive CS shunt & $9 \pm 2$ & $8 \pm 2$ & $8 \pm 2$ \\
\hline \multirow[t]{3}{*}{ LVEDP $(\mathrm{mm} \mathrm{Hg})$} & Ischemia & $8 \pm 2.9$ & $12 \pm 5.6$ & $12 \pm 5.5$ \\
\hline & CS shunt & $7 \pm 1.1$ & $8 \pm 1.0$ & $6 \pm 2.0$ \\
\hline & Occlusive CS shunt & $8 \pm 1.2$ & $8 \pm 1.6$ & $8 \pm 1.0$ \\
\hline \multirow[t]{3}{*}{ LVSWI ( $\%$ change) } & Ischemia & 100 & $67 \pm 26$ & $58 \pm 18$ \\
\hline & CS shunt & 100 & $66 \pm 34$ & $64 \pm 20$ \\
\hline & Occlusive CS shunt & 100 & $87 \pm 20$ & $82 \pm 15$ \\
\hline
\end{tabular}

$H R$, Heart rate; $C O$, cardiac output; $M A P$, mean arterial pressure; $L A P$, left atrial pressure; $L V E D P$, left ventricular end-diastolic pressure; $L V S W I$, left ventricular stroke work index; $C S$, coronary sinus.

onary artery occlusion, persisted throughout ischemia, and only gradually disappeared during reperfusion. Systolic shortening recovered to $36 \% \pm 24 \%$ after 60 minutes of reflow (Figure 2). Conversely, circumflex contraction presented no change during the ischemic-reperfusion interval.

Extended systolic contraction into diastole (diastolic dysfunction) persisted during the entire reperfusion interval, measuring $38 \% \pm 16 \%$ at 60 minutes of reflow (Figure 3).

In the CS shunt group, CS pressure increased from $9 \pm$ $1 \mathrm{~mm} \mathrm{Hg}$ at baseline to $49 \pm 12 \mathrm{~mm} \mathrm{Hg}$ with the selfinflated balloon and to $77 \pm 12 \mathrm{~mm} \mathrm{Hg}$ with the manually inflated balloon.

Bulging during ischemia persisted with the self-inflated retrograde cannula, whereas some active contraction was retained at the percentage of systolic shortening $(16 \% \pm 6 \%$ of baseline, $P<.01$ ) (Figure 2) when the manually inflated balloon was used. Ventricular fibrillation occurred in the CS-shunt groups in 5 of 6 hearts in each group, with no difference compared with the ischemic group, although 3 and 4 hearts in the non-occlusive and occlusive shunt groups, respectively, fibrillated only at reperfusion. Table 1 shows the hemodynamic variables, documenting a comparable approximate $20 \%$ decrease in cardiac output in both shunted groups but a slightly (but not statistically different) better maintenance of LVSWI (26\% vs $18 \%$ ) in the manually inflated retrograde shunt group. This retrograde perfusion allowed a lower LVEDP than in the groups with unprotected ischemia $(6 \pm 2 \mathrm{~mm} \mathrm{Hg}$ in the non-occlusive shunt group and $8 \pm 1 \mathrm{~mm} \mathrm{Hg}$ in the occlusive shunt group vs $12 \pm 7 \mathrm{~mm} \mathrm{Hg}$ in the unprotected ischemia group at 60 minutes, $P=.06$ )
Retrograde perfusion during ischemia improved recovery of the percentage of systolic shortening after reperfusion (Figure 2$)$ to a relatively comparable level $(\sim 50 \%) 15$ minutes after reflow in both the self-inflated and manually inflated CS cannula. This contrasts with less than $20 \%$ systolic shortening without retrograde perfusion. This improvement was sustained, recovering to approximately $60 \%$ systolic shortening at 1 hour in both shunted groups.

Although systolic contraction was detected during the first part of diastole in the CS shunt groups (Figure 2), the percentage of diastolic time was reduced to $22 \% \pm 22 \%$ with the non-occlusive CS shunt at 60 minutes and to $9 \% \pm$ 9\% with the occlusive retrograde shunt. These levels of diastolic dysfunction were less $(P<.05)$ than in untreated hearts at 60 minutes after reperfusion.

CS creatine kinase-MB was $59 \% \pm 33 \%$ greater than baseline in the untreated group compared with $56 \% \pm 51 \%$ in the non-occlusive CS shunt group and $37 \% \pm 7 \%$ in the occlusive CS shunt group (no statistical significance was found).

CD levels were similar $(34 \% \pm 16 \%, 32 \% \pm 15 \%)$ in the untreated and non-occlusive shunt groups, respectively, after 60 minutes of reperfusion but decreased $(24 \% \pm 3 \%)$ when the occlusive CS shunt was used $(P<.05)$.

NO levels in CS plasma decreased to $13 \% \pm 15 \%$ below baseline values without retrograde shunting after 30 minutes of reperfusion (Figure 4), whereas NO levels were maintained in the non-occlusive CS shunt group and increased to $9 \% \pm 8 \%$ at 30 minutes after reperfusion $(P<.05)$.

CS levels of ET-1 increased in all groups after ischemia. In untreated hearts, ET-1 increased $40 \% \pm 12 \%$ and $34 \% \pm$ 


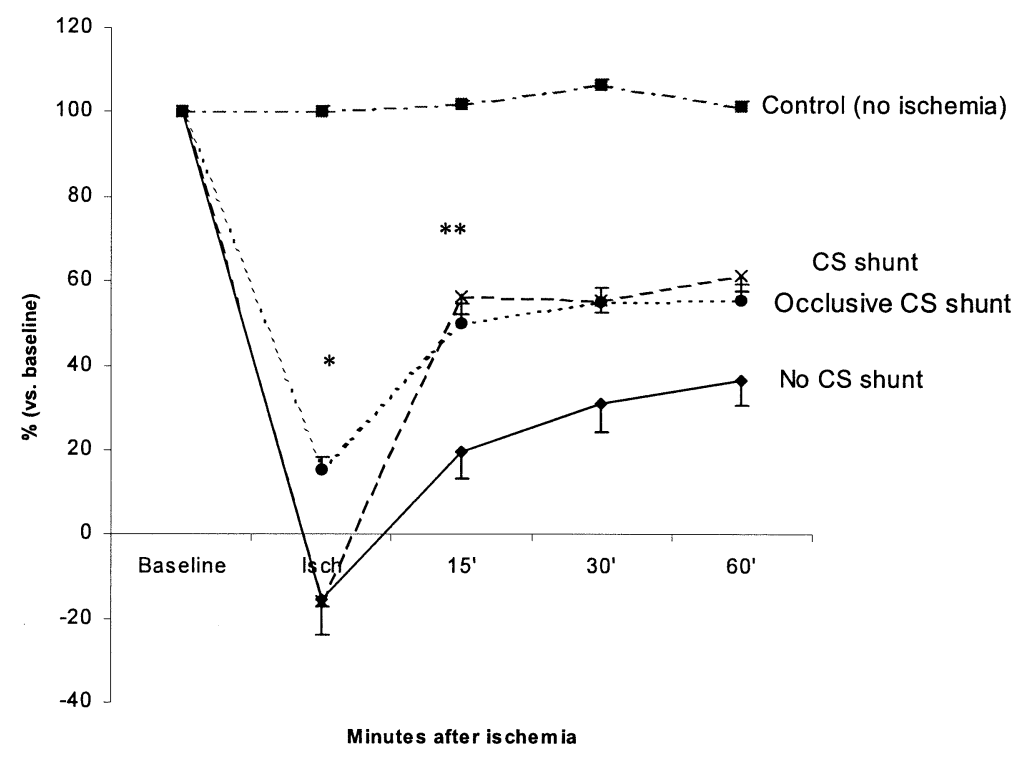

Figure 2. Systolic dysfunction: Percentage of contraction during systole versus baseline values in the different groups. Values expressed as mean \pm SEM. ${ }^{*} P<.01$, occlusive CS shunt vs non-occlusive CS shunt and ischemic groups; ${ }^{*} P<.05$, CS shunt groups vs ischemic group.) $C S$, Coronary sinus.

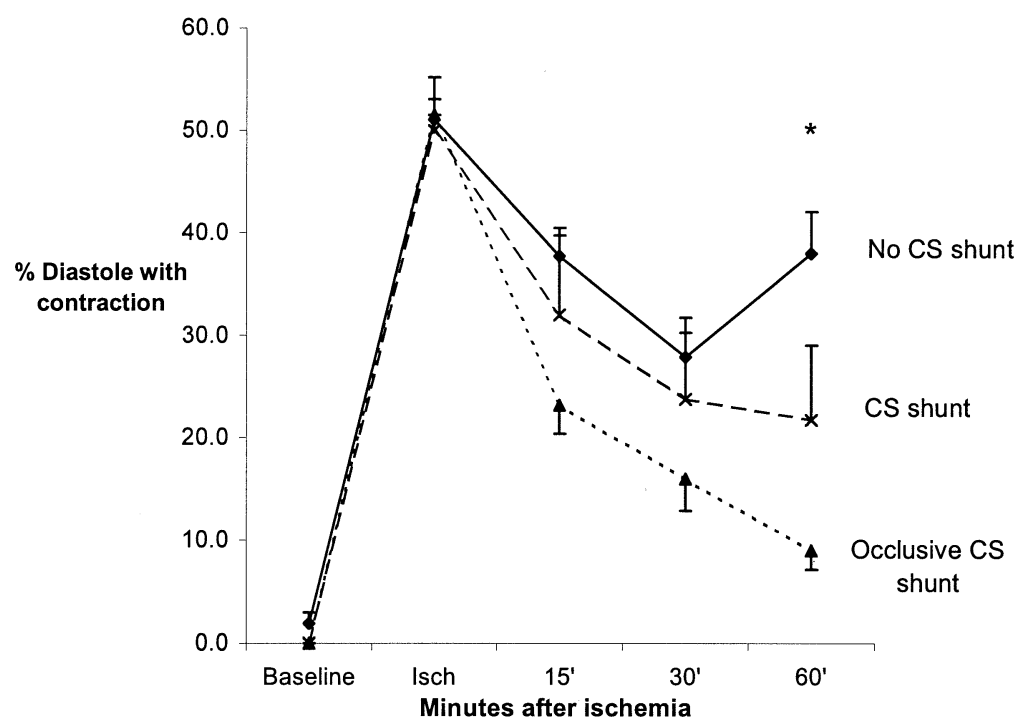

Figure 3. Diastolic dysfunction: Percentage of diastole in which contraction was detected. Values expressed as mean \pm SEM. ( ${ }^{*} P<.05$, occlusive CS shunt group vs control ischemia group). CS, Coronary sinus.

$16 \%$ at 30 and 60 minutes, respectively, compared with $41 \% \pm 17 \%$ and $32 \% \pm 15 \%$, respectively, in the nonocclusive CS shunt group and $31 \% \pm 5 \%$ and $24 \% \pm 3 \%$, respectively, in the occlusive CS shunt group.

\section{Discussion}

Reversible postischemic ventricular dysfunction, or myocardial "stunning," recently has been more implicated with interventional coronary reperfusion, especially during OP-
$\mathrm{CAB} .{ }^{8}$ Conversely, systems that maintain flow, such as intracoronary shunts, improve anterior wall motion after 15 minutes of LAD occlusion in the animal model. ${ }^{3}$ These perfusion methods differ from ischemic preconditioning, in which results are controversial, especially after repetitive ischemia. ${ }^{1,9}$

An alternate method is retrograde CS perfusion, which is currently a frequently used technique of delivering cardioplegic solutions during CABG. This study defines the 
benefit of this technique in limiting systolic and diastolic dysfunction after temporary coronary occlusion. This protective method is most effective for the left ventricle because of impaired right ventricular perfusion. ${ }^{10}$ Retrograde flow may be even more useful in beating heart surgery without bypass, because myocardial oxygen demands are high and cardioplegic protection is not possible.

The strategy for ensuring CS flow is important in the beating heart, because adequate perfusion pressure is needed to ensure nourishment during cardiac work. For example, a self-inflating retrograde cannula has an irregular wall allowing some leakage back into the right atrium, which can lessen CS pressure and possibly impair perfusion. For that reason, we tested both the self-inflating balloon (permitting some backflow into the right atrium) and manually inflating balloon (limiting backflow). We found a higher CS pressure (79 vs $42 \mathrm{~mm} \mathrm{Hg}$ ) and less diastolic dysfunction with the occlusive balloon. Retroperfusion during ischemia has been shown to be beneficial in previous reports. ${ }^{11-13}$ The importance of sustaining perfusion pressure in salvaging ischemic tissue is evident in experimental studies of retrograde venous perfusion during pressure-controlled intermittent coronary occlusion. ${ }^{11}$ The background for this method comes from clinical retroperfusion for circulatory support during failed percutaneous transluminal coronary angioplasty procedures as a rapid way to restore flow before emergency extracorporeal circulation and CABG. ${ }^{12}$ These reports led others to test this method successfully in patients undergoing $\mathrm{OPCAB},{ }^{13}$ and our study quantifies this benefit.

Fifteen minutes of regional no-flow ischemia is the classic method of inducing systolic stunning, as defined by Braunwald and Kloner. ${ }^{14}$ This is usually the time interval in which coronary artery flow is compromised during OPCAB. Simultaneously, such stunning leads to prolongation of systole into the diastolic interval, ${ }^{15}$ thus impairing relaxation and rendering the heart more turgid to limit suction and increasing end-diastolic pressure as the result of stiffening from this mechanism. Our studies codify such severe systolic and diastolic dysfunction in this swine model. Systolic dysfunction was evidenced by the persistent regional impaired contractility 60 minutes after reperfusion (Figures 5 and 6). Additionally, contraction extended into early diastole, thereby confirming that impaired postsystolic shortening is a marker of early myocardial stunning. ${ }^{16}$

Similar experimental models indicate that contractile dysfunction may last up to 24 hours postischemia. ${ }^{6}$ A consequence of such systolic dysfunction is that the prolonged contraction in the postischemic area can extend into early diastole. This prolonged contractile effect impairs ventricular compliance during the rapid filling phase and compromises suction for ventricular filling. Our studies confirmed this effect, because LVEDP increased in the untreated

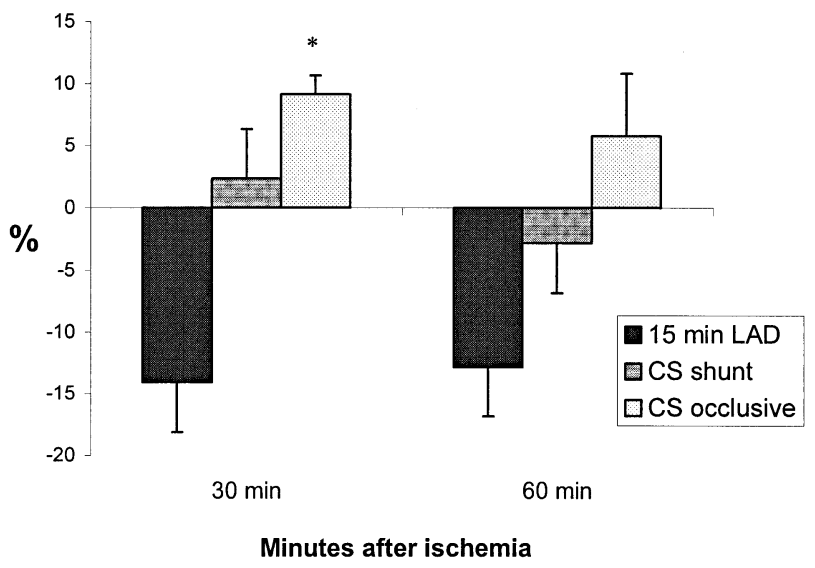

Figure 4. Percentage of baseline NO production in CS plasma at 30 and 60 minutes after ischemia. Values expressed as mean \pm SEM. ( ${ }^{*}<.05$ vs unprotected ischemia group). $L A D$, Left anterior descending; CS, coronary sinus.

hearts. Figure 6 shows that the reduced systolic contraction started later in the reperfused region, so that an excitationcontraction mismatch simultaneously developed along with prolongation of systolic contraction into diastole. The excitation-contraction uncoupling may be an important feature of myocardial stunning and may account for the late thickening reported postbypass in septal damage. ${ }^{17}$

Continuous retrograde perfusion during LAD occlusion significantly improved excitation-contraction mismatch and reduced systolic and diastolic dysfunction. We recognize that retrograde perfusion was not sufficient because ischemic-induced ventricular fibrillation occurred in shunted studies and contractile function did not return to normal after 60 minutes of reperfusion. Nevertheless, postischemic systolic shortening was significantly higher in retrograde perfused hearts than in control hearts. Moreover, the occlusive coronary shunt prevented bulging during ischemia and, more important, nearly obliterated diastolic dysfunction in comparison with the untreated and non-occlusive shunt groups. We suspect that the improved diastolic function, with prevention of extended contraction, caused the avoidance of increased LVEDP during reperfusion, a numeric reading that may be a marker of improved suction and greater compliance.

We suspect that endothelial protection may have been afforded by retrograde perfusion during ischemia, because release of NO was significantly higher in the retrograde perfusion group versus the untreated group. Further studies are needed to verify these hypotheses.

\section{Critique of the Experimental Model}

Our experimental design studied ischemia in juvenile pig hearts without coronary artery disease, so generalization of our data must be performed cautiously. Our model differs 


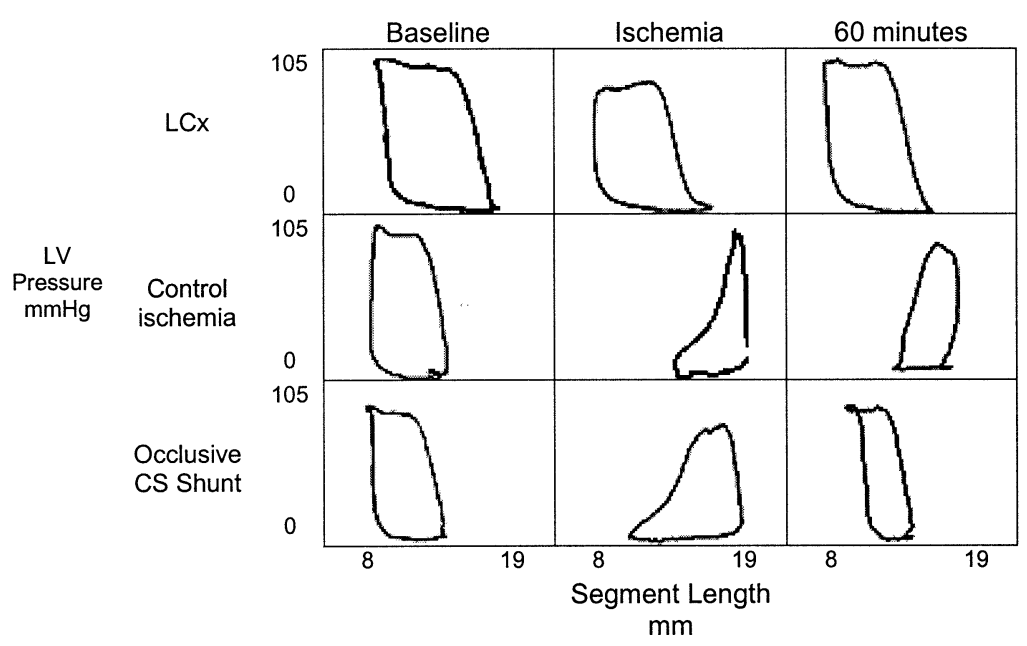

Figure 5. Pressure-segment length work loops in the anterior free wall of the left ventricle before ischemia, during 15 minutes of LAD occlusion, and after 15, 30, and 60 minutes of LAD reperfusion of control non-ischemic (left circumflex coronary artery), control ischemic (LAD), and occlusive shunt (LAD) groups. Note the shift of the loop to the right in the ischemic heart (indicating muscular distention) and the bent to the right (indicating dyskinesia). $L V$, Left ventricle; $C S$, coronary sinus; $L C x$, left circumflex coronary artery.

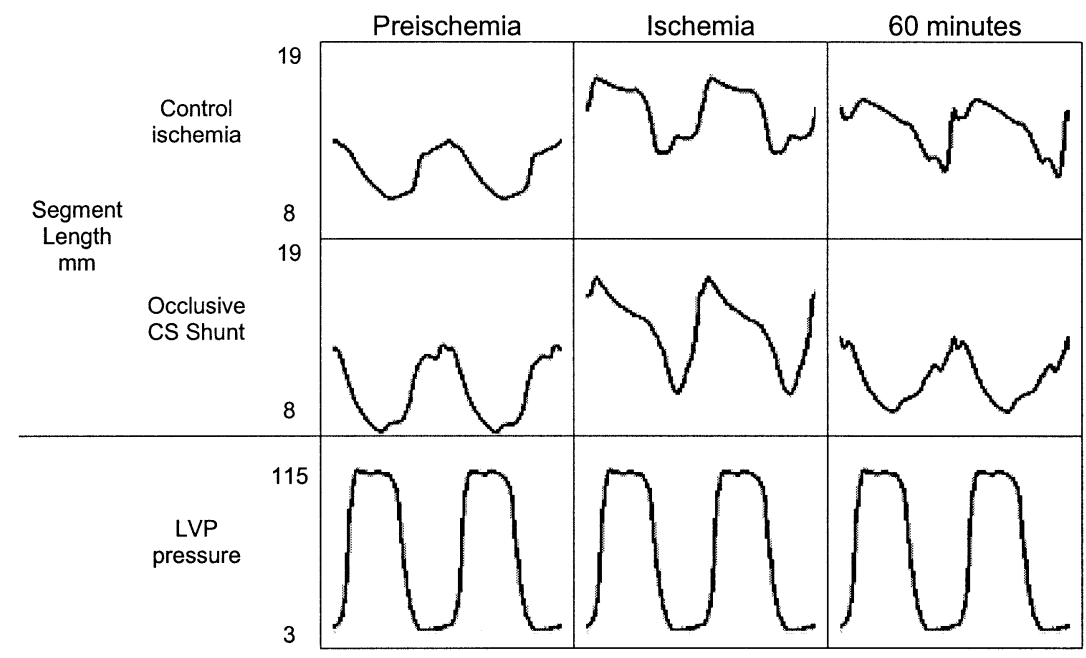

Figure 6. Raw tracings of myocardial segment lengths in control ischemia (LAD) and occlusive CS shunt (LAD) groups during 15 minutes of LAD occlusion and after 15 minutes, 30 minutes, and 60 minutes of LAD reperfusion. Note the elevation of the tracing in the ischemic heart during ischemia and reperfusion, indicating distention, poor contraction during systole, and extension of contraction into diastole. LVP, Left ventricular pressure; CS, coronary sinus.

from those including patients with extensive coronary artery disease, in whom collateral flow develops and limits the extent of ischemia after sudden LAD occlusion. Nevertheless, our method of retrograde perfusion may parallel patients with moderate single-vessel stenosis and limited collateral flow.

Retraction of the heart (as is performed in OPCAB) may impair CS catheter flow, mainly when it is performed by compression of the myocardial wall. We did not use retraction techniques in our study.

\section{Conclusion}

This swine model of 15 minutes of LAD occlusion in the beating heart produces a stunning equivalent that includes an excitation-contraction mismatch, impaired systolic shortening, and prolonged contraction into early diastole that 
compromises systolic ejection and diastolic filling. Retrograde perfusion through aortic-coronary shunt during temporal ischemia is a recognized method in bypass procedures that allows an easy and rapid perfusion system in the OP$\mathrm{CAB}$ procedure. Our evidence that such retroperfusion, especially with an occlusive shunt that significantly reduces excitation-contraction uncoupling, improves postischemic systolic and diastolic function may allow this known methodology of improving myocardial perfusion to become a useful adjunct to OPCAB surgery.

\section{References}

1. Park SW, Tang XL, Qiu Y, Sun JZ, Bolli R. Nisoldipine attenuates myocardial stunning induced by multiple coronary occlusions in conscious pigs and this effect is independent of changes in hemodynamics or coronary blood flow. J Mol Cell Cardiol. 1996;28:655-66.

2. Hacker TA, Renstrom B, Nellis SH, Liedtke AJ. Effect of repetitive stunning on myocardial metabolism in pig hearts. Am J Physiol Heart Circ Physiol. 1997;42:H1395-4402.

3. Dapunt OE, Raji MR, Jeschkeit S, Dhein S, Kuhn-Régnier F, Südkamp $\mathrm{M}$, et al. Intracoronary shunt insertion prevents myocardial stunning in a juvenile porcine MIDCAB model absent of coronary artery disease. Eur J Cardiothorac Surg. 1999;15:173-9.

4. Constantini C, Sampaolesi A, Serra CM, Pacheco G, Neuburger J, Conci E, et al. Coronary venous retroperfusion support during highrisk coronary angioplasty in patients with acute ischemic syndromes. Circulation. 1989;80:116-25.

5. Glower DD, Spratt JA, Snow ND, Kabas JS, Davis JW, Olsen CO, et al. Linearity of the Frank-Starling relationship in the intact heart: the concept of preload recruitable stroke work. Circulation. 1985;71:9941009.

6. Glower DD, Spratt JA, Kabas JS, Davis JW, Rankin JS. Quantification of regional myocardial dysfunction after acute ischemic injury. Am J Physiol. 1987;255:H85-93.

7. Lesnefsky EJ, Fennessey PM, Van Benthuysen KM, McMurtry IF, Travis VL, Horwitz LD. Superoxide dismutase decreases early reperfusion release of conjugated dienes following regional canine ischemia. Basic Res Cardiol. 1989;84:191-6.

8. Chang PP, Sussman MS, Conte JV, Grega MA, Schulman SP, Gerstenblith G, et al. Postoperative ventricular function and cardiac enzymes after on-pump versus off-pump CABG surgery. Am J Cardiol. 2002;89:1107-10.

9. Maczewski M, Beresewicz A. The role of adenosine and ATP-sensitive potassium channels in the protection afforded by ischemic preconditioning against the post-ischemic endothelial dysfunction in guinea-pig hearts. J Mol Cell Cardiol. 1998;30:1735-47.

10. Gates RN, Laks H. Retrograde cardioplegia. In: Karp RB, Laks H, Wechsler SA, editors. Advances in cardiac surgery. St. Louis: Mosby; 1998. pp. 115-35.

11. Geary GG, Smith G, Suehiro GT, Zeman C, Siu B, McNamara JJ. Quantitative assessment of infarct size reduction by coronary venous retroperfusion in baboons. Am J Cardiol. 1982;50:1424-30.

12. Güden M, Akpinar B, Bayindir O, Sagbas E, Sanisoglu I, Demiroglu C. Early myocardial resuscitation via coronary sinus perfusion. Asian Cardiovasc Thorac Ann. 2000;8:330-2.

13. Bedi HS, Suri A, Kalkat MS, Sengar BS, Mahajan V, Chawla R, et al. Global myocardial revascularization without cardiopulmonary bypass using innovative technique for myocardial stabilization and perfusion. Ann Thorac Surg. 2000;69:156-64.

14. Braunwald E, Kloner RA. The stunned myocardium. Prolonged postischemic ventricular dysfunction. Circulation. 1982;66:1146-9.
15. Takayama M, Norris RM, Brown MA, Armiger LC, Rivers JT, White HD. Postsystolic shortening of acutely ischemic canine myocardium predicts early and late recovery of function after coronary artery reperfusion. Circulation. 1988;78:994-1007.

16. Hosokawa H, Sheehan FH, Suzuki T. Measurement of postsystolic shortening to assess viability and predict recovery of left ventricular function after acute myocardial infarction. J Am Coll Cardiol. 2000; 35:1842-9.

17. Hirata N, Maeda S, Takiuchi S, Iwata K, Ohtake S, Sawa Y, et al. Real time assessment of myocardial revascularization during CABG by means of ultrasonic integrated backscatter. Eur J Cardiothorac Surg. 1999;16:156-9.

\section{Discussion}

Dr Y. Joseph Woo (Philadelphia, Pa). I know your group has published reports on the simultaneous use of antegrade and retrograde cardioplegia. In that setting you can control the exact pressures, antegrade and retrograde, to prevent myocardial edema. I was wondering if you looked at the tissue at all to see if there were any issues with myocardial edema?

The Emory group has done a lot with perfusion-assisted distal assist, and I was wondering if you could apply your idea to putting in a catheter and applying distal perfusion through a partial shunt?

Dr Castella. We provided a simultaneous flow rate for antegrade and retrograde perfusion in the past and did not see edema in those studies. Edema formation was not evaluated in the current experiments, but it is not expected with simultaneous perfusion, since coronary venous drainage is via the thebesian veins that empty into the ventricles.

We would be interested in seeing if our retrograde perfusion studies, done during ischemia, can amend the antegrade studies at Emory, which are done after the region is grafted.

Dr Nilas Young (Sacramento, Calif). It is the rare patient presenting for OPCAB with a single-vessel disease. Much more commonly, patients have multivessel disease and are at higher risk for major coronary artery occlusion during the case. Have you taken this model another step further to have a multidisease or preinfarction model, or do you plan on doing that?

Dr Castella. You are correct, but the results would be magnified with multivessel disease, since the systolic and diastolic dysfunction would be avoided in several areas. This refers to all other regions than segments perfused by the right coronary artery, where retrograde perfusion is limited, due to problems in distribution.

Dr Jakob Vinten-Johansen (Atlanta, $G a$ ). Can you achieve the same goal by just occluding the CS and then perfusing ostensibly with deoxygenated blood (CS blood), or do you need the arterial component of that? Because essentially you're admixing both venous coronary effluent and arterial blood.

Dr Castella. We cannot answer your question directly, but the preservation of systolic and diastolic function relates to oxygenated perfusion, since the coronary sinus $\mathrm{Po}_{2}$ is very low. Evidence of the oxygenated supply was shown when the artery was opened, as our previous use of internal thoracic artery anastomosis during regional ischemia showed bright red retrograde return coming from the distal vessel. 